semicarbazide as a trapping agent ${ }^{11}$; whereupon less than $0.2 \%$ of the original $\mathrm{C}^{14}$-activity was recovered as formaldehyde. It is rather improbable that this trace amount of formaldehyde could be the result of metabolic activity; and it is believed that it is due to autooxidation of some of the liberated methanol. These results indicate clearly the absence of the oxidative pathway: methanol $\rightarrow$ formaldehyde $\rightarrow$ formate $\rightarrow$ carbon dioxide. Paper chromatographic analysis revealed the presence of mono-methylated Dipterex as the only $\mathrm{C}^{14}$-metabolite (cf. 8) ; suggesting that the other $\mathrm{C}^{14}$-metabolite(s) are volatile substances.

11 H. Aebi and A. Hassan, Helv. chem. Acta 43, 544 [1960].
Based on previous studies, it may be concluded that the detoxification of the insecticide "in vivo" proceeds as follows:

a) In the rat: exclusive hydrolysis of the phosphonate bond of both Dipterex and its demethylated metabolite ${ }^{7}$.

b) In Prodenia larvae: $70 \%$ hydrolysis of $O$-methyl ester linkages ${ }^{6}$ and $30 \%$ splitting of $\mathrm{C}-\mathrm{P}$ bond.

c) In cotton plant: hydrolysis of the phosphonate bond, followed by demethylation of the methylated phosphates ${ }^{5}$.

d) In microorganisms: exclusive hydrolysis of $O$. methyl ester bond $(\mathrm{s})^{8}$.

\section{Über die Aufnahme verschiedener Zucker durch Chlorella pyrenoidosa}

\author{
W. Hülsen und U. Prenzel
}

Kernforschungszentrum Karlsruhe, Schule für Kerntechnik

(Z. Naturforschg. 21 b, 500-501 [1966] ; eingeg. am 15. Februar 1966)

Systematische Untersuchungen über heterotrophes Wachstum von Algen sind von $\mathrm{D}_{\text {ANFoRTH }}{ }^{1}$ referiert worden. In den Arbeiten wird mitgeteilt, daß sowohl Glucose wie Galaktose heterotrophes Wachstum verschiedener Algen (3 Chlorella-Arten, Scenedesmus und Prototheca) erhalten. Mit Mannose wachsen die 3 Chlorella Arten nicht. Bei Verwendung von Fructose ist es fraglich, ob das Wachstum von Chlorella pyrenoidosa und Chlorella ellipsoidae aufrechterhalten wird. Chlorella vulgaris wächst mit Fructose. Diese Untersuchungen verschiedener Autoren ${ }^{2}$ sind bei sehr unterschiedlichen Kulturbedingungen hinsichtlich Zusammensetzung des anorganischen Mediums, Belichtung, Begasung und Temperatur durchgeführt worden, und auch das „Wachstum" wird mit verschiedenen Maßstäben gemessen. Da die Stoffbewegung zwischen Medium und Zelle die Voraussetzung für die Ernährung und das Wachstum der Zelle ist, liegt die Vermutung nahe, daß die im Dunkeln von den Algen aufgenommene Menge Zucker ihrer Verwertbarkeit in der Zelle entspricht. Bei Untersuchungen über Stoffbewegung in Synchronkulturen von Chlorella pyrenoidosa haben wir die Menge verschiedener Zucker bestimmt, die in $3 \mathrm{Stdn}$. unter Lichtausschluß aufgenommen wird.

Die Synchronkultur von Chlorella pyrenoidosa erhielten wir nach folgendem Verfahren ${ }^{3}$ :

Wenige Zellen des Stammes 211/8b der Algensammlung des Pflanzenphysiologischen Instituts der Universität Göttingen wurden in das von $\mathrm{KuHL}^{4}$ beschriebene Nährmedium gebracht und in einem Thermostaten

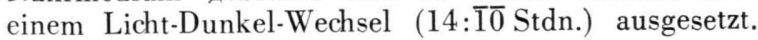

1 W. F. Danforth, in: Physiology and Biochemistry of Algae, ed. R. A. Lewin, Acad. Press, New York 1962.

2 A. C. Neish, Can. J. Botany 29, 68 [1951] ; H. Samejima u. J. Myers, J. gen. Microbiol. 18, 107 [1958]; H. A. Barker, J. cellular comparat. Physiol. 7, 73 [1935].
Die Temperatur betrug $30 \pm 0,1{ }^{\circ} \mathrm{C}$. Die Belichtung von rund $20000 \mathrm{Lux}$ in den Kulturgefäßen wurde durch Philips-Leuchtstoffröhren vom Typ TL 20/32 und TL 20/55 erzielt. Die Kultur wurde ständig mit Luft begast (20 l pro Stde.), der 1,5\% $\mathrm{CO}_{2}$ beigemischt war. Nach $48 \mathrm{Stdn}$. wurde die Kultur auf etwa $10^{6} \mathrm{Zellen} / \mathrm{ml}$ verdünnt. Nach einem weiteren Licht-Dunkel-Wechsel war die Zellzahl ungefähr auf das 20-fache angestiegen. Die Kultur wurde am Ende der Dunkelphase mit Nährmedium auf $10^{7}$ Zellen $/ \mathrm{ml}$ eingestellt. Zu dieser Synchronkultur wurden $0,5 \mu \mathrm{Mol} / \mathrm{ml}$ des ${ }^{14} \mathrm{C}$-markierten Zuckers $\left(0,1\right.$ bis $1,0 \mu \mathrm{Ci}{ }^{14} \mathrm{C} / \mathrm{ml}$ Medium) gegeben und die Algen weitere $3 \mathrm{Stdn}$. im Dunkeln bei gleichbleibender Begasung im Thermostaten belassen. Nach dieser Zeit wurden die Algen durch Filtration vom Medium getrennt und gewaschen. Aliquote Teile der Algen und des Filtrats wurden in $\mathrm{O}_{2}$-Atmosphäre verbrannt und das gebildete $\mathrm{CO}_{2}$ in Monoäthanolamin absorbiert. Die Radioaktivität dieser Lösungen wurde im Flüssig-Szintillationszähler bestimmt ${ }^{5}$. Die Ergebnisse sind in der Tabelle zusammengefaßt.

\begin{tabular}{|l|r|c|c|c|}
\hline $\begin{array}{c}\text { eingesetzte } \\
\begin{array}{c}\text { C-14-mar- } \\
\text { kierteZucker }\end{array}\end{array}$ & \multicolumn{4}{|c|}{ \% der eingesetzten Radioaktivität } \\
\hline D-Glucose & 13 & $72(63-84)^{2}$ & 9 & $10(6-14)$ \\
in den Algen & A & im Filtrat \\
D-Mannose & 9 & $58(42-66)$ & 6 & $19(8-35)$ \\
D-Fructose & 8 & $27(19-32)$ & 5 & $60(54-67)$ \\
D-Galaktose & 4 & $<2$ & 3 & $>96$ \\
D-Ribose & 4 & $<2$ & 3 & $>96$ \\
D-Arabinose & 3 & $<2$ & 3 & $>96$ \\
\hline
\end{tabular}

Tab. 1. A = Anzahl der Versuche. ${ }^{2}$ Mittelwerte (mit Schwankungsbreiten).

Aus der Tabelle ist ersichtlich, daß die Kohlenstoffatome von Glucose, Mannose und Fructose die Zell-

3 A. Pirson u. H. Lorenzen, Z. Bot. 46, 53 [1958].

4 A. Kuhl u. H. Lorenzen, in: Methods in Cell Physiology, Bd. 1, S. 159, Prescott, London 1964.

5 F. Kalberer u. J. Rutschmann, Helv. chim. Acta 44, 1956 [1961]. 
wand passiert haben und metabolisiert worden sind. Ob Galaktose, Ribose und Arabinose ebenfalls angereichert wurden, läßt sich nicht entscheiden, da die Fehlerbreite unserer Aktivitätsbestimmung im Filtrat $\pm 3 \%$ beträgt und die geringe Aktivität in den Algen auch als unspezifische Adsorption der Zucker an den Algen gedeutet werden kann.

Wir danken Herrn Dipl.-Phys. Gamber vom Lichttechnischen Institut der TH Karlsruhe für die Eichung des Luxmeters.

\section{Membranfragmente mit unterschiedlichem Verhältnis von Chlorophyll a und Chlorophyll b nach Ultraschall-Fragmentierung von Spinat- Chloroplasten}

\section{B. BIEHL}

Botanisches Institut der Tierärztlichen Hochschule Hannover

(Z. Naturforschg. 21 b, 501-502 [1966] ; eingeg. am 6. Februar 1966)

Für die Beurteilung der Funktion und Struktur der Thylakoide wird unter anderem auch die Bestimmung der Chlorophylle nach Fragmentierung der Chloroplasten herangezogen. Nach Lichtenthaler und Calvin ${ }^{1}$ zeigen die kleinsten Membranfragmente, die bei der Fraktionierung von Thylakoidsystemen nach Ultraschallbehandlung anfallen, das gleiche Verhältnis von Chlorophyll a zu Chlorophyll b wie intakte Chloroplasten von Spinat. Das Verhältnis $(a: b)$ war innerhalb $6 \%$ identisch. Dieser Befund stützt die Annahme, daß es sich bei diesen Fragmenten um Aggregate funktionell und morphologisch gleichartiger Einheiten (Quantasomen ${ }^{2}$ ) der Membranen handelt. [Spinatchloroplasten in hypertonischer Saccharose isoliert, in hypotonischem Medium beschallt und die Fragmente durch Differential-Zentrifugation fraktioniert: 1. 10 Min. $20000 \mathrm{~g}$; 2. $30 \mathrm{Min} .145000 \mathrm{~g}$ (Quantasomenaggregate); chlorophyllfreier Überstand.] Demgegenüber fanden Boardman und Anderson ${ }^{3}$ nach Fragmentierung durch Digitonin sehr unterschiedliche Verhältnisse von Chlorophyll $\mathrm{a}$ und $\mathrm{b}$ und nehmen zwei verschiedene Typen von Partikeln an, von denen einer, mit hohem Gehalt an Chlorophyll a, gelöst wird. [Spinatchloroplasten in isotonischer Saccharose isoliert, in hypotonischem Medium mit 0,5\% Digitonin fragmentiert und durch Differential-Zentrifugation fraktioniert: $(\mathrm{a}: \mathrm{b}$ in Klammern) : ganze Chloroplasten (3,0); 10 Min. 1000 g $(2,4)$; 30 Min. $10000 \mathrm{~g}(2,5)$; 30 Min. $50000 \mathrm{~g}(4,6)$; 60 Min. $144000 \mathrm{~g}(6,2)$; chlorophyllhaltiger Überstand $(5,4)$.

Als eine Konsequenz in der Gegenüberstellung der Ergebnisse der zitierten Arbeiten ergibt sich, daß Digitonin als Fragmentierungsmittel prinzipiell anders auf die Thylakoide wirkt (partielle Lösung ${ }^{3}$ ) als Ultra-

1 H. K. Lichtenthaler u. M. Calvin, Biochim. biophysica Acta [Amsterdam] 79, 30 [1964].

2 R. B. Park u. N. G. Pon, J. molecular Biol. 3, 1 [1961].

3 N. K. Boardman u. J. A. Anderson, Nature [London] 203, 166 [1964].

4 B. BieHL, Planta 67, 265 [1965].

5 Mod. Meth. Pflanzenanalyse Bd. IV, S. 159, Springer-Verlag, Berlin-Göttingen-Heidelberg 1955. schall (Trennung von Partikeln identischer Zusammensetzung ${ }^{1}$ ) und diese qualitativ unterschiedliche Wirkung in der Analyse der Fragmente nach Trennung durch Differential-Zentrifugation zum Ausdruck kommt. Die hier mitgeteilten Untersuchungen zeigen jedoch, daß Ultraschall-Desintegration zumindest in bezug auf die Relation von Chlorophyll a und b nicht zu Membranfragmenten einheitlicher Zusammensetzung führt:

Aus isolierten, einmal gewaschenen Chloroplasten $\left(\mathrm{C}_{1}\right)$ wurden die Membrankörper durch hypotonischen Schock freigelegt. Diese Suspensionen oder die aus ihnen durch quantitative Sedimentation des Chlorophylls und Resuspension gereinigten Membrankörper (,Partikel“) wurden beschallt und fraktioniert zentrifugiert (Tab. 1). (Nähere Angaben zu Material und Methode ${ }^{4}$.) Die mit Aceton extrahierten Chlorophylle wurden nach Überführung in Äther photometrisch aus den Extinktionen bei $\lambda=643 \mathrm{~m} \mu$ und dem Maximum zwischen $\lambda=661$ und $663 \mathrm{~m} \mu$ bestimmt und nach Smith und Benitez ${ }^{5}$ berechnet ${ }^{4}$. Die Reproduzierbarkeit des Bestimmungsverfahrens (a:b) wurde an jeweils zwei getrennt isolierten Chloroplasten-Fraktionen innerhalb von 22 Aufarbeitungen geprüft: Standardabw. $= \pm 3 \%$ (relativ), Streubereich der Einzelbestimmungen für $99 \%$ Sicherheit $= \pm 9$ Prozent $^{6}$. Diese Bestimmung war besser reproduzierbar als nach Trennung der Chlorophylle an Dünnschichten ${ }^{7,8}$ oder Saccharose-Säulen ${ }^{9,10}$. Alle Aufarbeitungen erfolgten bei 0 bis $+5^{\circ} \mathrm{C}$ unter gleichen Bedingungen. Das a:b-Verhältnis in $\mathrm{C}_{1}$-Chloroplasten zeitlich verschiedener Aufarbeitungen war veränderlich, vermutlich auf Grund vorgegebener Unterschiede des Blattmaterials. Darum sind Fraktionen nur innerhalb der einzelnen Aufarbeitungen vergleichbar. Nach Ultraschallbehandlung ganzer Chloroplasten (Aufarb. 1-7, Tab. 1) zeigten die Sedimente I und II ein um 7-11\% gleichförmig vermindertes, die Sedimente III und die Überstände mit einer Ausnahme ein unterschiedlich erhöhtes a:b-Verhältnis. Für „Partikel“ gilt prinzipiell das gleiche. Auch Messungen der photosynthetischen Aktivität brachten Unterschiede solcher Fraktionen ${ }^{11}$. Für das Mengenverhältnis der Chlorophylle ergibt sich ein faßbarer Unterschied von $12-22 \%$ erst aus dem Vergleich der Sedimente I und II einerseits und III andererseits, dagegen nicht bei

6 K. Doerffel, Beurteilung von Analysenverfahren und Ergebnissen, Springer-Verlag, Berlin-Göttingen-Heidelberg 1962.

7 K. EgGer, Planta 58, 664 [1962].

8 A. Hager u. T. Bertenrath, Planta 58, 564 [1962].

9 A. BAUER, Planta 51, 84 [1958].

10 H. FALK, Planta 51, 49 [1958].

11 G. J Асові, unveröffentlichte Ergebnisse. 